\title{
Previsión del ambiente térmico para el ganado lechero mediante redes neuronales
} artificiales

\section{Forecasting the thermal environment for dairy cattle by means of artificial neural networks}

DOI: $10.53499 /$ sfjeasv1n4-001

Received in: August 1st, 2021

Accepted in: September 30th, 2021

\author{
Pedro Hurtado de Mendoza Borges \\ Doctor en Máquinas Agrícolas por la Universidad de Rostock, Alemania \\ Institución: Universidad Federal de Mato Grosso \\ Dirección: Av. Fernando Corrêa da Costa nº 2367, Boa Esperança, Cuiabá - MT, Brasil \\ E-mail: pedrohmborges14@gmail.com \\ Zaíra Morais dos Santos Hurtado de Mendoza \\ Doctora en Ciencia Forestal por la Universidad Federal de Viçosa \\ Institución: Universidad Federal de Mato Grosso \\ Dirección: Av. Fernando Corrêa da Costa nº 2367, Boa Esperança, Cuiabá - MT, Brasil \\ E-mail: zairamorais09@gmail.com \\ Pedro Hurtado de Mendoza Morais \\ Estudiante de Agronomía de la Universidad Federal de Mato Grosso \\ Institución: Universidad Federal de Mato Grosso \\ Dirección: Av. Fernando Corrêa da Costa nº 2367, Boa Esperança, Cuiabá - MT, Brasil \\ E-mail: pedromorais08@ hotmail.com
}

\section{RESUMEN}

El presente estudio tuvo como objetivo la previsión del ambiente térmico para el ganado lechero mediante redes neuronales artificiales, de acordó con la temperatura y humedad diaria. En la investigación se utilizaron los valores diarios de esas variables, disponibles en el Instituto Nacional de Meteorología de Brasil. Los datos correspondieron a las series históricas registradas en estaciones convencionales con tiempo de operación superior a 30 años hasta 2020. A continuación, se seleccionaron los municipios Canarana, Matupá, Nova Xavantina y Santo Antônio de Leverger, localizados en Mato Grosso, Brasil. Con base en los dados climatológicos, se estimó el Índice de Temperatura y Humedad diario en el calendario Juliano. Posteriormente, se probaron 35 arquitecturas de redes neuronales artificiales con tipología perceptrón de múltiples camadas, siendo la variable de entrada el día Juliano y la de salida el Índice de Temperatura y Humedad. La idoneidad de las redes fue verificada por el coeficiente de determinación, el error absoluto medio, el error cuadrático medio, el porciento medio del error absoluto y la normalidad de los residuos. No hubo diferencias entre los valores estimados por las redes y los obtenidos a partir de las series históricas. La rede de mejor desempeño y eficiencia para cada municipio, también fue comprobada por el análisis gráfico de los residuos. Se concluyó que las redes neuronales con tipología perceptron de dos camadas ocultas fueron apropiadas en el pronóstico del ambiente térmico natural para el ganado lechero.

Palabras-claves: Bienestar animal, construcciones rurales, inteligencia artificial. 


\begin{abstract}
The present study aimed to forecast the thermal environment for dairy cattle through artificial neural networks, according to the daily temperature and humidity. The research used the daily values of these variables, available in the National Institute of Meteorology of Brazil. The data corresponded to the historical series registered in conventional stations with an operating time of more than 30 years until 2020. Next, the municipalities Canarana, Matupá, Nova Xavantina and Santo Antônio de Leverger, located in Mato Grosso, Brazil, were selected. Based on the climatological data, the Temperature and Humidity Index was determined for each day of the year in the Julian calendar. Subsequently, 35 artificial neural network architectures with multiple layer perceptron typology were tested, the input variable being the Julian day and the output variable being the Temperature and Humidity Index. The suitability of the networks was verified by the coefficient of determination, the mean absolute error, the mean square error, the mean percentage of the absolute error and the normality of the residuals. There were no differences between the values estimated by the networks and those obtained from the historical series. The network with the best performance and efficiency for each municipality was also verified by the graphic analysis of the residuals. It was concluded that the neural networks with perceptron typology of two hidden layers were appropriate in the forecast of the natural thermal environment for dairy cattle.
\end{abstract}

Keywords: Animal welfare, rural buildings, artificial intelligence.

\title{
1 INTRODUCCIÓN
}

La reducción significativa em el desempeño productivo y reproductivo de los animales por causa del estrés térmico ha sido objetivo principal de diversos estudios (BERTONCELLI et al., 2013; MANTECA et al., 2013; VIANA et al., 2013; SILVA et al., 2016; FIALHO et al., 2018; OLIVEIRA et al. 2018). Entre otros índices, el conforto térmico animal puede ser evaluado mediante el Índice de Temperatura y Humedad (ITH), propuesto por Thom (1959). Ese índice tiene como ventaja, con relación a los demás, su fácil cálculo a partir de las variables climáticas temperatura y humedad, disponibles en los bancos de datos meteorológicos o que pueden ser medidas en las propias instalaciones utilizándose sensores. Luego, esos resultados pueden servir como referencia para la toma de decisiones con el propósito de amenizar el estrés por calor en los animales.

Por otro lado, el manejo adecuado de animales requiere el conocimiento previo de las condiciones ambientales para garantizar el confort y poder evitar o minimizar las pérdidas causadas por el estrés térmico. La previsión de variables meteorológicas puede ser efectuada por medio de diversos recursos, entre los cuales se destacan las redes neurales artificiales. Esos artificios computacionales son flexibles y robustos ante las amplias fluctuaciones e inestabilidades del microclima, bien como tienen elevada capacidad para el reconocimiento y repetición de padrones. Esas propiedades fueron comprobadas en los estudios realizados por Bilgili \& Sahin (2010), Wu et al. (2010), Yasar et al. (2012), Depiné et al. (2013) y Borges et al. (2018, 2019, 2021a). 
Con base en las investigaciones consultadas se deduce que las redes neuronales artificiales pueden ser una útil y poderosa herramienta computacional para el procesamiento de datos meteorológicos, siendo su uso promisor en la previsión de la temperatura y humedad del aire a partir de las series históricas registradas en estaciones meteorológicas. Luego, este trabajo se fundamenta en la hipótesis de que los referidos recursos pueden suministrar las informaciones necesarias para caracterizar el ambiente térmico en las proximidades de esas estaciones, lo que puede contribuir en la toma de decisiones con la finalidad de conducir el manejo de los animales adecuadamente. La problemática presentada motivó este estudio, que tiene como objetivo la previsión del ambiente térmico para el ganado lechero mediante redes neuronales artificiales, conforme la temperatura y humedad del aire para cada día del año.

\section{MATERIAL Y MÉTODOS}

En la investigación se utilizaron los valores medios diarios de la temperatura del aire y de la humedad relativa, disponibles en el Banco de Datos Meteorológicos para la Enseñanza e Investigación (BDMEP) del Instituto Nacional de Meteorología (INMET), correspondientes a series históricas con elevado número de registros hasta el año 2020. La selección de las Estaciones se realizó de acuerdo con el tiempo de operación superior a 30 años hasta 2020 con máximo de $15 \%$ de fallas durante los registros y la diferencia de altitud, latitud o longitud. Considerándose los citados criterios, se seleccionaron las estaciones meteorológicas localizadas en Canarana, Matupá, Nova Xavantina y Santo Antônio de Leverger, Estado de Mato Grosso, Brasil.

En la Tabla 1 se presentan los principales datos de las estaciones meteorológicas convencionales seleccionadas para este estudio. De forma general, el clima del Estado de Mato Grosso fue clasificado como Aw (PEEL et al., 2007; ALVARES et al., 2013). La temperatura media anual varia de $22^{\circ} \mathrm{C}$ a $27,6^{\circ} \mathrm{C}$, con máximas entre $28^{\circ} \mathrm{C}$ a $33,3^{\circ} \mathrm{C}$ y mínimas de $16^{\circ} \mathrm{C}$ a $20^{\circ} \mathrm{C}$, registrándose las menores temperaturas al sureste del estado. La pluviosidad media oscila entre $1200 \mathrm{~mm}$ y $2200 \mathrm{~mm}$, ocurriendo los mayores valores al norte del estado (RAMOS et al., 2017; INMET, 2021).

Tabla 1. Principales datos de las estaciones meteorológicas convencionales utilizadas en la investigación.

\begin{tabular}{lccccc}
\multicolumn{1}{c}{ Municipio } & Código OMM & Período & \multicolumn{2}{c}{ Coordenadas $\left(^{\circ}\right)$} & $\begin{array}{c}\text { Altitud } \\
(\mathrm{m})\end{array}$ \\
\cline { 4 - 6 } & & & Latitud & Longitud & \\
\hline Canarana & 83270 & $1987-2020$ & $-13,47$ & $-52,27$ & 430 \\
Matupá & 83214 & $1985-2020$ & $-10,19$ & $-54,95$ & 272 \\
Nova Xavantina & 83319 & $1987-2020$ & $-14,70$ & $-52,35$ & 316 \\
Santo Antônio de Leverger & 83364 & $1986-2020$ & $-15,78$ & $-56,07$ & 140 \\
\hline
\end{tabular}


Fuente: Adaptado por los autores de INMET (2021).

Los municipios con estaciones meteorológicas seleccionados para este trabajo se representaron en la Figura 1. La referida Figura se elaboró a partir de bases cartográficas continuas cedidas por el Instituto Brasilero de Geografía y Estadística (IBGE), con auxilio del programa QGIS (IBGE, 2021; QGIS, 2020).

Figura 1. Municipios seleccionados para el estudio y su localización geográfica en Mato Grosso, Brasil.

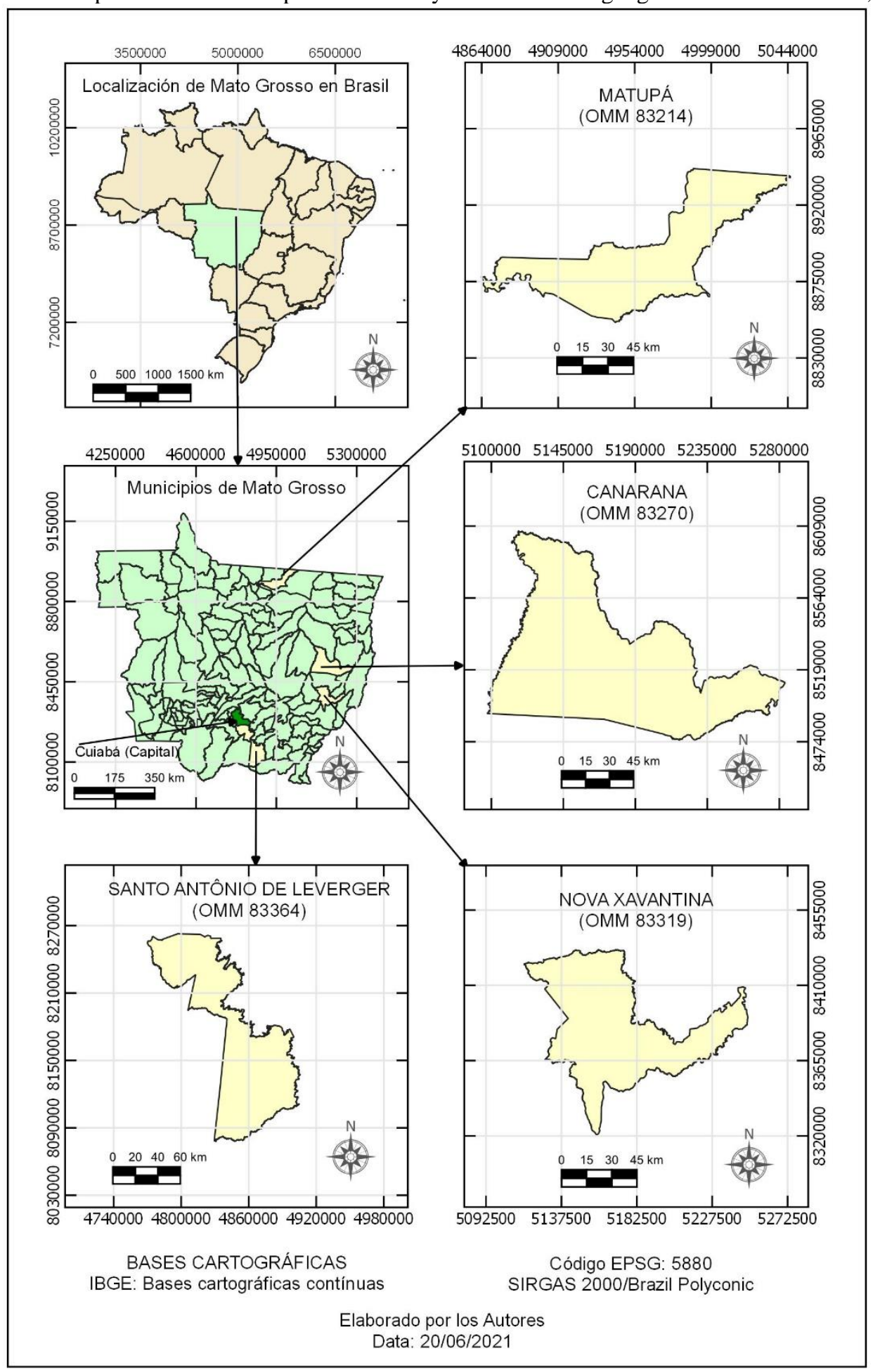


Los valores obtenidos para las variables temperatura y humedad relativa del aire se almacenaron en planillas electrónicas EXCEL. Después, se utilizaron las funciones de filtro y clasificación para detectar y eliminar posibles fallas ocurridas durante el registro, de las informaciones climáticas. Esas operaciones permitieron la selección de los datos válidos para estimar el Índice de Temperatura y Humedad (ITH) correspondiente al día Juliano, conforme la ecuación propuesta por Thom (1959), dada por:

$\mathrm{ITH}=\mathrm{T}_{\mathrm{bs}}+0,36 \cdot \mathrm{T}_{\mathrm{po}}+41,5$

en que:

ITU = Índice de Temperatura y Humedad (adimensional);

$\mathrm{T}_{\mathrm{bs}}=$ Temperatura del aire $\left({ }^{\circ} \mathrm{C}\right)$;

$\mathrm{T}_{\mathrm{po}}=$ Temperatura del rocío $\left({ }^{\circ} \mathrm{C}\right)$.

Por otro lado, la temperatura del rocío se determinó por:

$$
\mathrm{T}_{\mathrm{po}}=\frac{1}{\frac{1}{\mathrm{~T}_{\mathrm{bs}}+273,15}-\frac{\ln \frac{\mathrm{UR}}{100}}{5417}}-273,15
$$

en que:

$\mathrm{T}_{\mathrm{po}}=$ Temperatura del rocío $\left({ }^{\circ} \mathrm{C}\right)$;

$\mathrm{T}_{\mathrm{bs}}=$ Temperatura del aire $\left({ }^{\circ} \mathrm{C}\right)$;

$\mathrm{UR}=$ Humedad relativa del aire $(\%)$.

Los valores del Índice de Temperatura y Humedad (ITH) correspondientes al día Juliano se introdujeron en el programa R. Luego, se procesaron por medio de la función "neuralnet" del referido programa para obtener diversas arquitecturas de redes neuronales artificiales (R CORE TEAM, 2020). En este estudio se optó por la tipología perceptron de múltiples camadas (MPL), para la previsión del Índice de Temperatura y Humedad a partir del día Juliano. En seguida, se definieron varias estructuras de redes, teniendo como variable independiente en la camada de entrada el día Juliano y como variable dependiente en la camada de salida el Índice de Temperatura y Humedad.

Con la finalidad de elevar la eficiencia y la precisión de las redes se evaluaron arquitecturas con una y dos camadas intermediarias, conteniendo hasta 25 neuronas por camada. Además, las variables de entrada y salida se normalizaron entre 0 y 1 para evitar la saturación y probables errores en los resultados. Después, los valores estimados fueron desnormalizados para expresarlos en la escala original, aplicándose las relaciones: 


$$
\begin{aligned}
& V_{\text {nor }}=\frac{V_{\text {ori }}-V_{\text {min }}}{V_{\text {max }}-V_{\text {min }}} \\
& V_{\text {dnor }}=V_{\text {nor }} \cdot\left(V_{\text {max }}-V_{\text {min }}\right)+V_{\text {min }}
\end{aligned}
$$

en que:

$\mathrm{V}_{\text {nor }}=$ Valor normalizado (adimensional);

$\mathrm{V}_{\mathrm{dnor}}=$ Valor desnormalizado (adimensional);

$\mathrm{V}_{\text {ori }}=$ Valor original del conjunto de dados (adimensional);

$\mathrm{V}_{\min }=$ Valor mínimo del conjunto de dados (adimensional);

$\mathrm{V}_{\max }=$ Valor máximo del conjunto de dados (adimensional).

Los dados fueron separados de forma aleatoria en dos conjuntos. En el primero se incluyeron $75 \%$ de los valores y el segundo $25 \%$, siendo destinados a la elaboración y a la validación de las redes, respectivamente. En todas las arquitecturas se utilizó la función logística para activar las redes, generándose eventualmente los pesos iniciales entre $-0,5$ y 0,5. Las camadas se interconectaron por sinopsis completas y orientadas solamente al frente ("feed forward"), es decir, cada neurona de la camada i se combinó con todas las neuronas de la camada i+1. También, se aplicó la retro propagación del error ("back propagation") como algoritmo de conocimiento. Los índices de aprendizaje y momento fueron, respectivamente, de 0,2 y 0,8. Se definió como criterio de finalización un error inferior a 0,05. Como los valores de la variable dependiente variaron entre 0 y 1 , se optó por la función sigmoide para la transferencia en la camada de salida. Los procedimientos descritos se realizaron con el programa $\mathrm{R}(\mathrm{R}$ CORE TEAM, 2020).

Con el propósito de reducir el número de redes neuronales obtenidas y seleccionar las más eficientes y precisas, se determinaron el coeficiente de determinación $\left(\mathrm{R}^{2}\right)$, el error absoluto medio (MAE), el error cuadrático medio (MSE), la raíz del error cuadrático medio (RMSE) y el porciento medio del error absoluto (MAPE). Las redes con mejores resultados fueron analizadas mediante las pruebas Kolmogorov-Smirnov, Lilliefors y Shapiro-Wilk para verificar la normalidad de los residuos, bien como se aplicó la prueba de t-Student para evaluar la calidad del ajuste entre los valores observados y estimados.

Otros criterios analíticos adoptados fueron el coeficiente de correlación, el índice de concordancia de Willmott, bien como el índice de desempeño, resultante del producto de los anteriores para medir la proximidad entre valores estimados y observados. Además, se determinó el coeficiente de Nash-Sutcliffe (NASH \& SUTCLIFFE, 1970). Aunque, los criterios presentados permiten inferir sobre la precisión de 
las redes en la previsión del Índice de temperatura y Humedad, en este estudio se evaluó el comportamiento de los residuos gráficamente con el objetivo de verificar la existencia de tendencias inadecuadas en su distribución. Luego, se elaboraron el histograma de residuos, el diagrama cuantilcuantil con las bandas del intervalo de confianza, el gráfico de dispersión con los residuos para las variables dependiente e independiente y el diagrama de dispersión para cada rede neuronal artificial satisfactoria correspondiente al municipio, en función del día juliano.

\section{RESULTADOS Y DISCUSIÓN}

Durante la investigación se probaron 35 tipologías de redes neuronales artificiales, presentando una camada de entrada y otra de salida, siendo divididas en dos grupos. En el primer grupo se incluyeron 10 combinaciones con una camada intermediaria, variando el número de neuronas entre 5 y 15 . El segundo grupo poseía dos camadas intermediarias, conteniendo entre 5 y 9 neuronas cada una, formando las 25 composiciones restantes. Las arquitecturas con dos camadas intermediarias tuvieron mejores resultados, razón por la cual fueron seleccionadas para el posterior análisis de desempeño y precisión.

En la Tabla 2 se encuentran los valores de los índices correspondientes a la rede neuronal satisfactoria para cada municipio. De acuerdo con esa Tabla, los índices fueron semejantes, indicando que la idoneidad de las redes para la previsión del ambiente térmico en los cuatro municipios fue equivalente. Los valores del coeficiente de determinación $\left(\mathrm{R}^{2}\right)$ fueron superiores al valor mínimo aceptable de 0,7 para un ajuste adecuado entre los dados observados y estimados, bien como el porcentaje del error absoluto medio (MAPE) fue inferior a 10\%, aunque se prefiere que sea menor a 5\%. Las restantes métricas para la evaluación preliminar de ajuste fueron apropiadas.

Tabla 2. Valores de los índices correspondientes a la rede neuronal satisfactoria para cada municipio.

\begin{tabular}{|c|c|c|c|c|c|c|c|}
\hline \multicolumn{2}{|c|}{ Estación meteorológica } & \multirow{2}{*}{$\begin{array}{l}\text { Tipología } \\
\text { (MLP) }\end{array}$} & \multirow{2}{*}{$\begin{array}{l}\mathrm{R}^{2} \\
(-)\end{array}$} & \multirow{2}{*}{$\begin{array}{c}\text { MAE } \\
(-)\end{array}$} & \multirow{2}{*}{$\begin{array}{c}\text { MSE } \\
(-)\end{array}$} & \multirow{2}{*}{$\begin{array}{c}\text { RMSE } \\
(-)\end{array}$} & \multirow{2}{*}{$\begin{array}{c}\text { MAPE } \\
(\%)\end{array}$} \\
\hline Municipio & Código & & & & & & \\
\hline Canarana & 83270 & $1-5-8-1$ & 0,7132 & 0,0742 & 0,0085 & 0,0922 & 7,6958 \\
\hline Matupá & 83214 & $1-6-9-1$ & 0,7018 & 0,0763 & 0,0091 & 0,0954 & 8,0554 \\
\hline Nova Xavantina & 83319 & $1-9-8-1$ & 0,7114 & 0,0751 & 0,0087 & 0,0933 & 7,8036 \\
\hline Santo Antônio de Leverger & 83364 & $1-7-5-1$ & 0,7293 & 0,0717 & 0,0082 & 0,0916 & 7,1923 \\
\hline
\end{tabular}

Según las pruebas de Kolmogorov-Smirnov, Shapiro-Wilk y Lilliefors aplicadas a los residuos de las redes neuronales artificiales para los cuatro municipios, existen evidencias de normalidad en su distribución, pues las probabilidades fueron superiores a 0,05 (Tabla 3). También, en la Tabla 3, se observa que no hubo diferencias significativas entre los valores observados y los estimados por las cuatro redes, 
conforme la prueba de hipótesis ( $\mathrm{t}$-Student), lo que se comprueba por la elevada probabilidad ( $\mathrm{p}>0,05)$. Esos resultados constituyen la premisa básica para un adecuado ajuste entre los valores del Índice de Temperatura y Humead (ITU), calculados a partir de los dados climáticos del INMET y los obtenidos por medio de las redes neuronales artificiales.

Tabla 3. Probabilidad de significancia para las pruebas aplicadas a la RNA de cada estación meteorológica.

\begin{tabular}{|c|c|c|c|c|c|c|}
\hline \multicolumn{2}{|c|}{ Estación meteorológica } & \multirow{2}{*}{$\begin{array}{l}\text { Tipologia } \\
\text { (MLP) }\end{array}$} & \multicolumn{3}{|c|}{ Pruebas de normalidad } & \multirow{2}{*}{$\begin{array}{l}\text { Prueba de } \\
\text { hipótesis } \\
\text { (Student) }\end{array}$} \\
\hline Municipio & Código & & $\begin{array}{l}\text { Kolmogorov- } \\
\text { Smirnov }\end{array}$ & $\begin{array}{l}\text { Shapiro- } \\
\text { Wilk }\end{array}$ & Lilliefors & \\
\hline Canarana & 83270 & $1-5-8-1$ & $\begin{array}{c}0,4722 \\
\text { (ns) }\end{array}$ & $\begin{array}{c}0,4012 \\
(\mathrm{~ns})\end{array}$ & $\begin{array}{c}0,8347 \\
\quad(n s)\end{array}$ & $\begin{array}{l}0,7251 \\
\quad(n s)\end{array}$ \\
\hline Matupá & 83214 & $1-6-9-1$ & $\begin{array}{c}0,6439 \\
(\mathrm{~ns})\end{array}$ & $\begin{array}{l}0,1855 \\
\quad(n s)\end{array}$ & $\begin{array}{l}0,5231 \\
\quad(\mathrm{~ns})\end{array}$ & $\begin{array}{l}0,8127 \\
\quad(\mathrm{~ns})\end{array}$ \\
\hline Nova Xavantina & 83319 & $1-9-8-1$ & $\begin{array}{l}0,3545 \\
\quad(\mathrm{~ns})\end{array}$ & $\begin{array}{c}0,3729 \\
(\mathrm{~ns})\end{array}$ & $\begin{array}{l}0,1158 \\
\quad(n s)\end{array}$ & $\begin{array}{l}0,9306 \\
(\mathrm{~ns})\end{array}$ \\
\hline Santo Antônio de Leverger & 83364 & $1-7-5-1$ & $\begin{array}{l}0,9130 \\
(\mathrm{~ns})\end{array}$ & $\begin{array}{c}0,8295 \\
\quad(n s)\end{array}$ & $\begin{array}{c}0,6268 \\
(\mathrm{~ns})\end{array}$ & $\begin{array}{c}0,9958 \\
\text { (ns) }\end{array}$ \\
\hline
\end{tabular}

Los coeficientes e índices de desempeño y eficiencia determinados para las redes neuronales artificiales de los cuatro municipios se resumen en la Tabla 4. Con base en esa Tabla, se puede confirmar un idóneo desempeño y una excelente eficiencia, una vez que los coeficientes de Nash Sutcliffe fueron muy elevados. Esos resultados indican que las redes neuronales artificiales fueron apropiadas para estimar el Índice de Temperatura y Humedad (ITH) de cada municipio, conforme el día Juliano, posibilitando la previsión del ambiente térmico para las vacas lecheras durante el año.

Tabla 4. Resultados sobre el desempeño y la eficiencia de la RNA de cada estación meteorológica.

\begin{tabular}{|c|c|c|c|c|c|c|}
\hline \multicolumn{2}{|c|}{ Estación meteorológica } & \multirow{2}{*}{$\begin{array}{l}\text { Tipologia } \\
\text { (MLP) }\end{array}$} & \multicolumn{3}{|c|}{ Principales Coeficientes } & \multirow{2}{*}{$\begin{array}{l}\text { Eficiencia } \\
\quad \text { (Nash } \\
\text { Sutcliffe) }\end{array}$} \\
\hline Municipio & Código & & Correlación & Willmott & Producto & \\
\hline Canarana & 83270 & $1-5-8-1$ & 0,8537 & 0,7594 & 0,6483 & 0,9308 \\
\hline Matupá & 83214 & $1-6-9-1$ & 0,8695 & 0,7689 & 0,6686 & 0,9237 \\
\hline Nova Xavantina & 83319 & $1-9-8-1$ & 0,8749 & 0,7435 & 0,6505 & 0,9351 \\
\hline Santo Antônio de Leverger & 83364 & $1-7-5-1$ & 0,8822 & 0,7690 & 0,6784 & 0,9648 \\
\hline
\end{tabular}

Con base en los resultados de las pruebas analíticas fue confirmada la normalidad de los residuos correspondientes a las redes neuronales artificiales de cada municipio, las cuales presentaron satisfactorio ajuste, desempeño y eficiencia. Sin embargo, el análisis gráfico permite visualizar con mayor clareza a distribución de los residuos referente a las suposiciones de normalidad e independencia, bien como la ocurrencia y la magnitud de valores discrepantes de los observados. Así, se elaboraron los histogramas de 
la densidad de probabilidad correspondientes a los residuos estandarizados para las redes neuronales artificiales de cada municipio (Figura 2).

Figura 2. Histogramas de la densidad de probabilidad correspondientes a los residuos estandarizados para las redes neuronales artificiales de cada municipio.

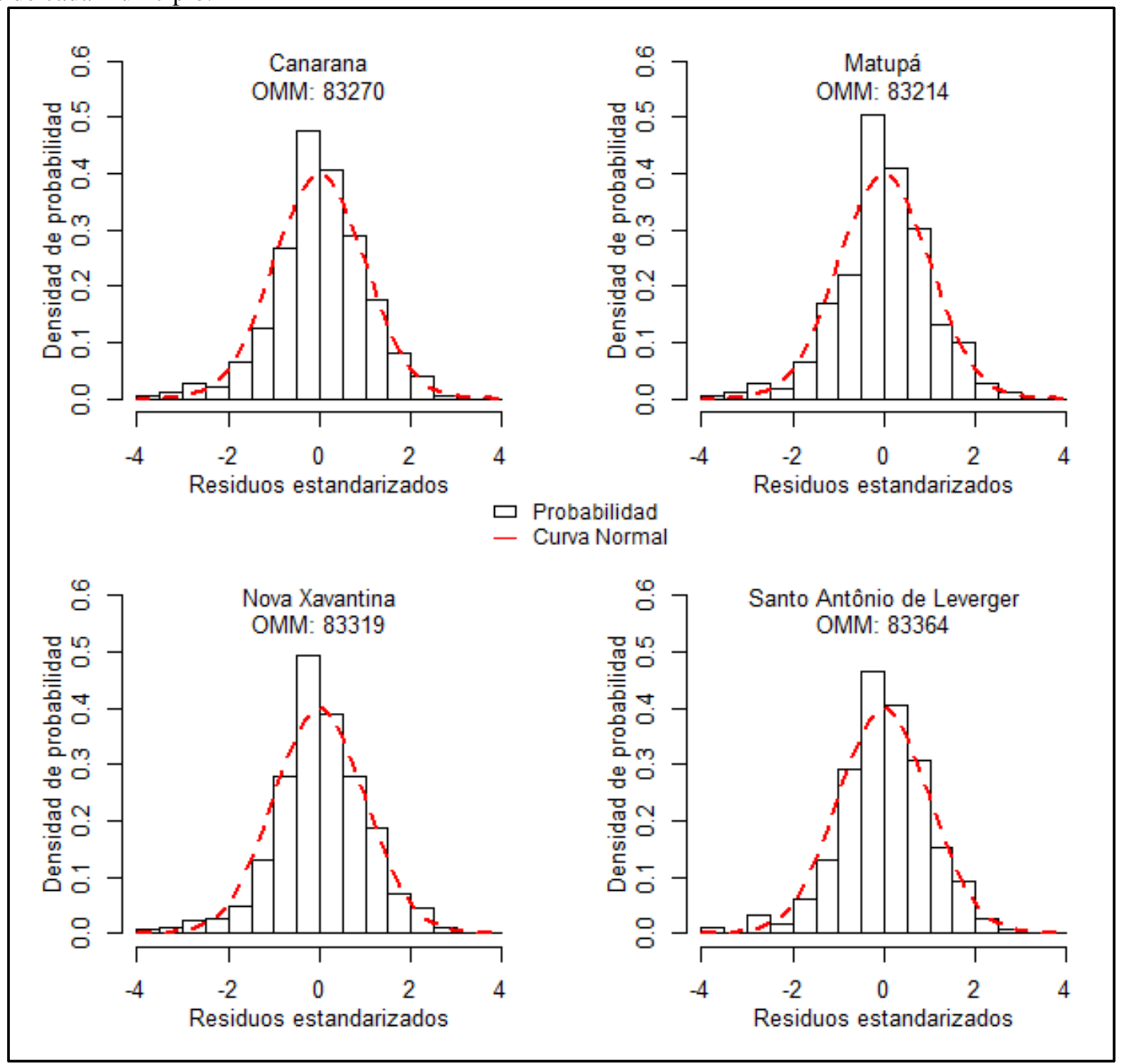

En la secuencia se confeccionaron los gráficos de probabilidad normal para los residuos estandarizados con las bandas del intervalo de confianza de $95 \%$, relativos a las redes neuronales artificiales de cada municipio (Figura 3). En la Figura se puede observar que la mayor parte de los puntos coordenados formados por los valores de los cuantiles teóricos y de los residuos se aproximaron a la línea de referencia o bisectriz, mientras en los extremos muy pocos se alejaron. También, se puede constatar que todos los puntos se mantuvieron entre los límites de confianza con 95\% del intervalo principal de -2 a 2. Las características descritas indicaron que los residuos evidenciaron distribución normal, lo que confiere mayor confiabilidad a la previsión del Índice de Temperatura y Humedad, obtenido por las redes desarrolladas. 
Figura 3. Gráficos de probabilidad normal correspondientes a los residuos estandarizados con las bandas del intervalo de confianza de $95 \%$ para las redes neuronales artificiales de cada municipio.
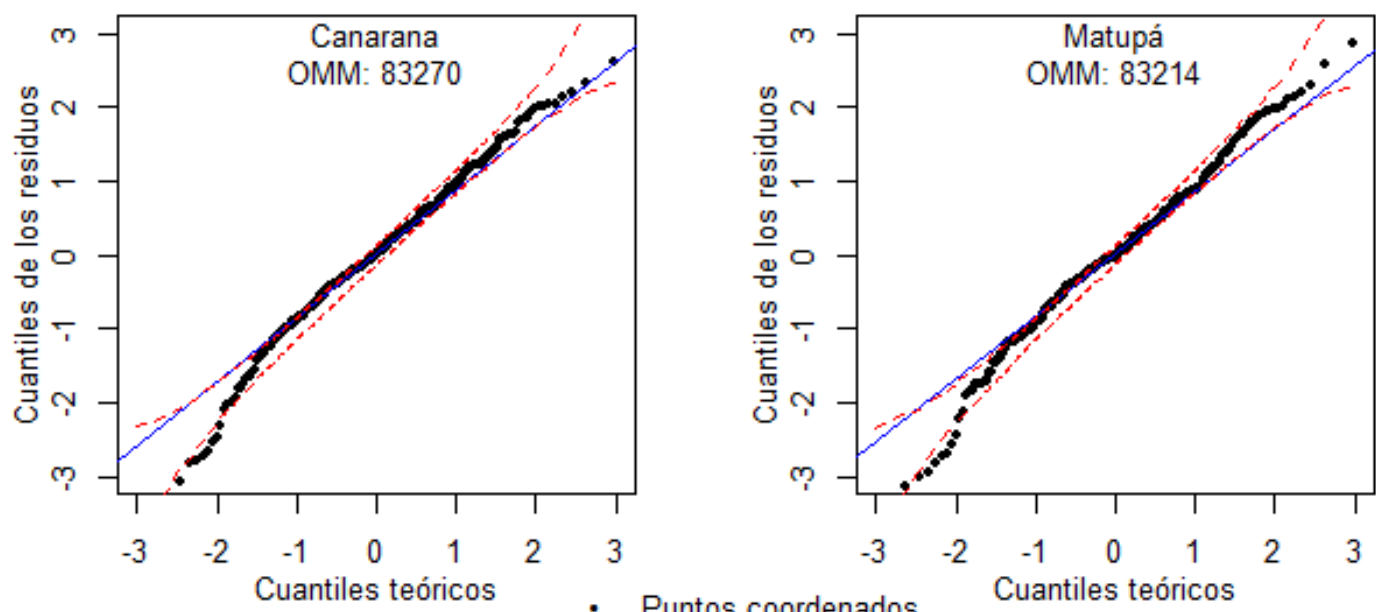

- Puntos coordenados

-.. Límites de confianza
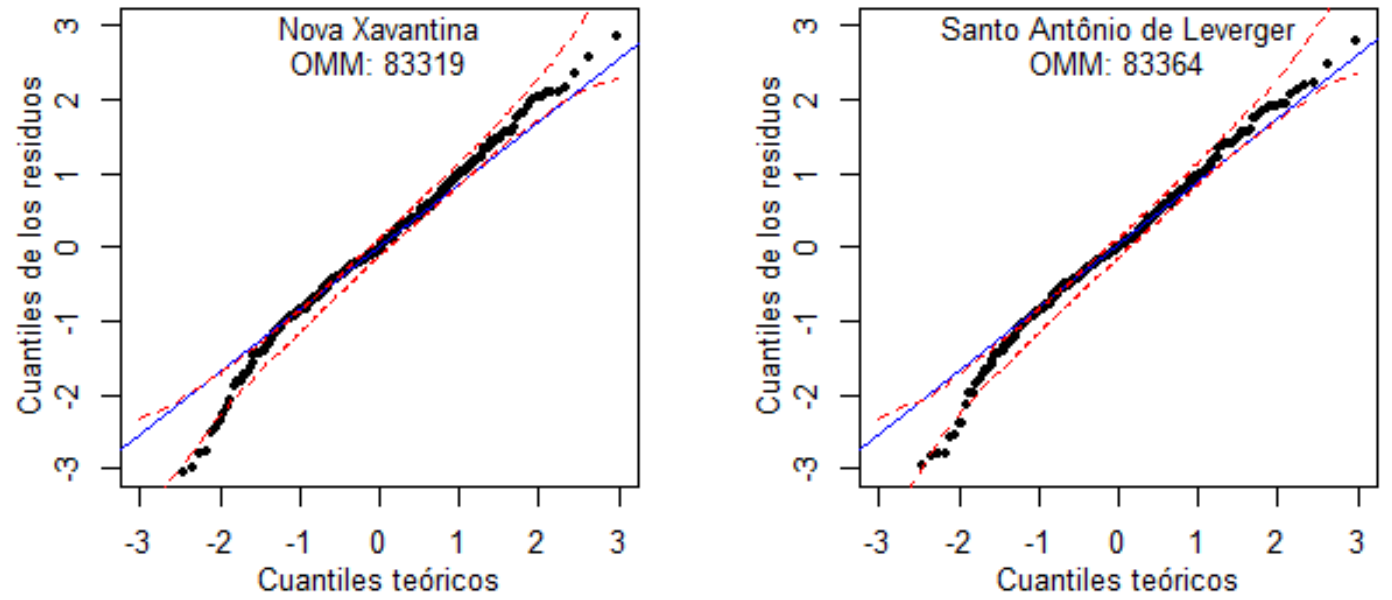

Complementando el análisis gráfico de los residuos para validar la suposición de su independencia, de acuerdo con el orden de colecta de los dados reales observados, se implementaron los diagramas de las Figuras 4 y 5. En esos diagramas se representaron las dispersiones de los residuos estandarizados, en función del día Juliano y del Índice de Temperatura y Humedad estimado por las redes neuronales de cada municipio. También, en las Figuras se incluyeron dos líneas horizontales en las ordenadas -2 y 2, lo que facilita identificar y cuantificar los puntos con los mayores desvíos. 
Figura 4. Dispersión de los residuos estandarizados correspondientes a las redes neuronales artificiales de cada municipio, en función del día Juliano.

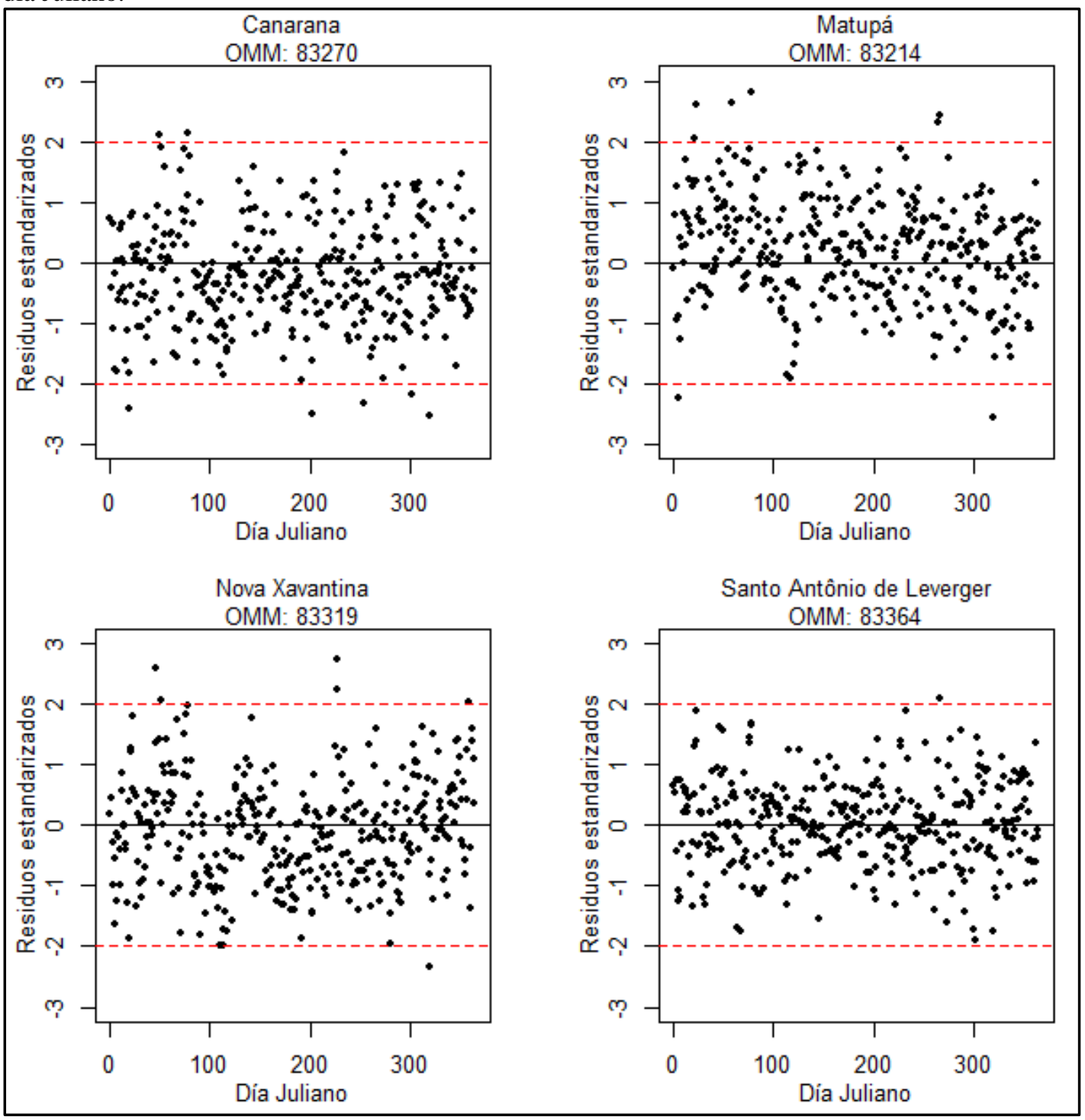

En las Figuras 4 y 5 se puede verificar que, a mayoría de los residuos se encuentran localizados próximos a la línea horizontal con de valor 0 , concentrándose principalmente en el intervalo de -2 a 2 y siendo muy pocos los restantes puntos. Aún, en esas Figuras se constata la ausencia de secuencias de residuos positivos y negativos, bien como no hubo evidencias con padrones de alternancia de signos, es decir, los residuos se distribuyeron aleatoriamente a lo largo de la línea horizontal, alrededor del valor 0 . Las características satisfactorias de la referida distribución confirmaron la independencia de los residuos, en función del orden de colecta, lo que corroboró la elevada eficiencia de las redes obtenidas para estimar con adecuada precisión el Índice de Temperatura y Humedad relativo a los cuatro municipios. 
Figura 5. Dispersión de los residuos estandarizados correspondientes a las redes neuronales artificiales de cada municipio, en función de los valores estimados del Índice de Temperatura y Humedad (ITH).
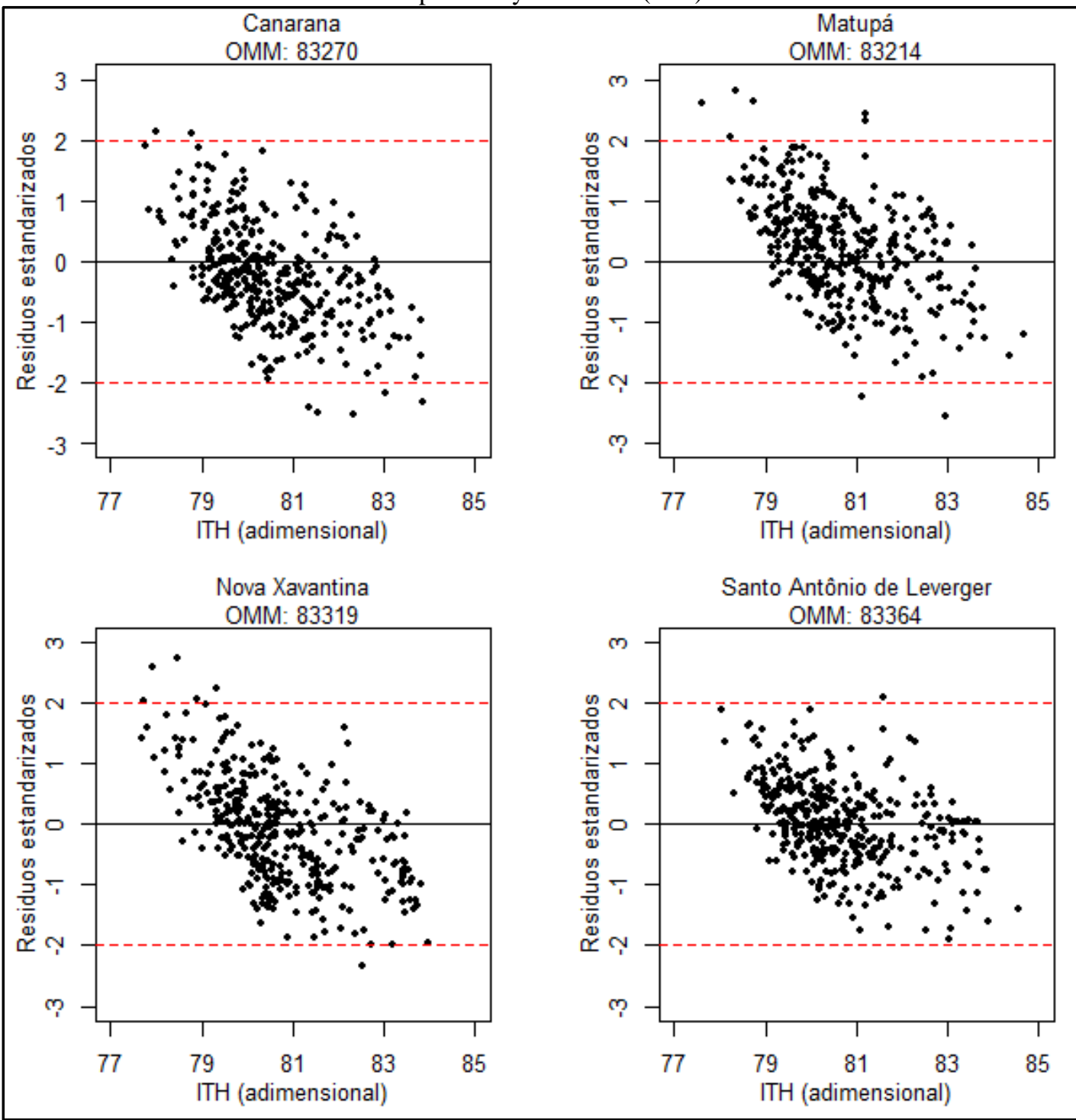

Después de confirmar el adecuado ajuste con satisfactorio desempeño y eficiencia de las redes neuronales artificiales mediante los procedimientos analíticos y gráficos, se confeccionaron los diagramas de dispersión con los valores del Índice de Temperatura y Humedad observados y estimados, en función del día Juliano (Figura 6). En esa Figura se verifica que, los valores estimados por las redes neuronales correspondientes a los cuatro municipios acompañaron la tendencia presentada por los valores reales observados para el mencionado índice. Así, se considera que las cuatro redes representaron de forma apropiada la previsión del ITH para los respectivos municipios. 
Figura 6. Dispersión de los valores observados del Índice de Temperatura y Humedad con las curvas de valores estimados por las redes neuronales artificiales de los cuatro municipios, en función del día Juliano.

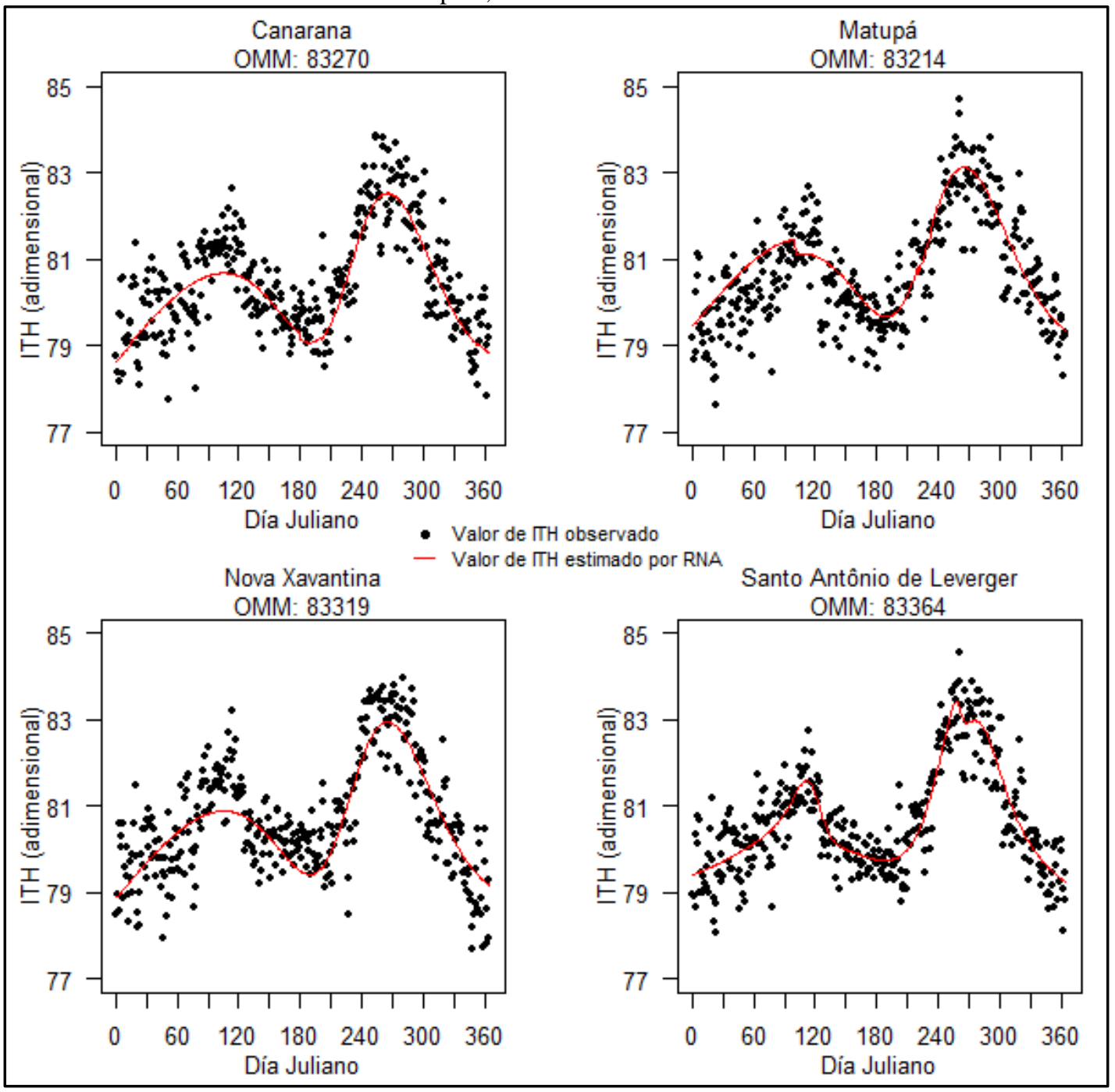

Con base en la Figura 6, se constató que los valores del Índice de Temperatura y Humedad fueron superiores a 78 durante todo el año para los cuatro municipios, inclusive se alcanzaron valores mayores que 82, principalmente a partir de la segunda quincena del mes de agosto hasta la primera quincena de octubre con valores extremos oscilando entre 83 y 84 en el mes de septiembre. De acuerdo con la escala propuesta por Thom (1959), los valores del Índice de Temperatura y Humedad entre 78 y 82 caracterizan situación de peligro, es decir, el animal se encuentra totalmente estresado reduciendo su producción significativamente. El referido autor afirmó que valores superiores a ese intervalo indican estado de emergencia, pudiendo causar la muerte del animal.

En esta investigación se ha demostrado la utilidad de las redes neuronales para auxiliar a los productores en la adopción de medidas dirigidas a garantizar el bienestar de las vacas lecheras durante su manejo. Los resultados obtenidos evidenciaron la excelente capacidad de las redes neuronales para estimar los valores del Índice de Temperatura y Humedad con adecuada precisión, aunque los dados climáticos 
utilizados en el proceso computacional presentaron elevada variabilidad para los cuatro municipios. También, se debe destacar que las referidas redes detectaron satisfactoriamente la estacionalidad del ambiente térmico en la región de estudio, aspecto de relevante importancia para el manejo de vacas, principalmente en confinamiento.

Los resultados del presente trabajo han comprobado que las condiciones térmicas naturales en los cuatro municipios investigados no fueron favorables para el desarrollo exitoso del ganado lechero. Luego, los proyectos de instalaciones para el manejo de vacas lecheras requieren especial atención, con relación a las coberturas y la climatización, lo que puede llevar a costos extras y elevadas inversiones. En ese sentido, las salas de ordeña deben tener prioridad para amenizar el estrés del animal antes de la retirada de la leche, siendo altamente recomendado el control y accionamiento automático de ventiladores con humidificadores a partir del monitoreo constante del ambiente térmico. Destinado a los pequeños y medios agricultores, Borges et al. (2021b) desarrollaron un prototipo de sistema automatizado de bajo costo para monitorear el Índice de Temperatura y Humedad en salas de ordeña, el cual permite accionar los referidos equipamientos hasta alcanzar el confort adecuado.

\section{CONCLUSIÓN}

Las redes neuronales artificiales, perceptron de múltiples camadas, formadas por tipología de dos camadas ocultas fueron apropiadas para estimar el Índice de Temperatura y Humedad, como función del día del año en el calendario Juliano. Las redes presentaron adecuada confiabilidad y eficiencia en las predicciones, lo que justifica su aplicación para auxiliar en la toma de decisiones referente al planeamiento, gestión y manejo de las vacas lecheras, principalmente confinadas. Además, las citadas redes permitieron clasificar el ambiente térmico en condiciones naturales, bien como identificar el período más crítico del año para los cuatro municipios estudiados.

\section{AGRADECIMIENTOS}

Al Instituto Nacional de Meteorología (INMET) por permitir la consulta a los dados de las estaciones climáticas convencionales de los municipios Canarana, Matupá, Nova Xavantina y Santo Antônio de Leverger, situados en el Estado de Mato Grosso, Brasil. Al Instituto Brasilero de Geografía y Estadística (IBGE) por la permisión al acceso de las bases cartográficas continuas. 


\section{REFERENCIAS}

ALVARES, C. A.; STAPE, J. L.; SENTElHAS, P. C.; GONÇALVES, J. L. M.; SPAROVEK, G. Köppen's climate classification map for Brazil. Meteorologische Zeitschrift, Stuttgart, v.22, n.6, p.711728, 2013.

DOI: http://dx.doi.org/10.1127/0941-2948/2013/0507

BERTONCELlI, P.; MARTIN, T. N.; ZIECH, M. F.; PARIS, W.; CELLA, O. S. Conforto térmico alterando a produção leiteira. Enciclopédia Biosfera, Goiânia, v.9, n.17, p.762-777, 2013.

BILGILI, M.; SAHIN, B. Comparative analysis of regression and artificial neural network models for wind speed prediction. Meteorology and Atmospheric Physics, v.109, n.1, p.61-72, 2010.

BORGES, P. H. M.; MENDOZA, Z. M. S.; MORAIS, P. H. M.; SANTOS, R. L. Artificial neural networks for predicting animal thermal comfort. Engenharia Agrícola, Jaboticabal, v.38, n.6, p.844856, 2018.

DOI: https://doi.org/10.1590/1809-4430-Eng.Agric.v38n6p844-856/2018

BORGES, P. H. M.; MENDOZA, Z. M. S.; MORAIS, P. H. M. Redes neurales artificiales para estimar las pérdidas en la producción lechera. Archivos de Zootecnia, Córdova, v.68, n.262, p.206-212, 2019.

BORGES, P. H. M.; CAVALCANTE, C. E.; MORAIS, P. H. M.; MENDOZA, Z. M. S. Monitoramento do ambiente térmico em salas de ordenha. Brazilian Journal of Animal and Environmental Research, Curitiba, v.4, n.2, p. 2796-2808, 2021 a.

DOI: http://doi.org/10.34188/bjaerv4n2-100

BORGES, P. H. M.; MENDOZA, Z. M. S.; MORAIS, P. H. M. Pronóstico anual de la carga térmica radiante aplicándose inteligencia artificial. Nativa, Sinop, v. 9, n. 3, p. 229-235, 2021b. DOI: https://doi.org/10.31413/nativa.v9i3.10122

DEPINÉ, H.; CASTRO, N. M. R.; PEDROLLO, O. C. Incertezas no preenchimento de falhas de chuvas horárias com redes neurais artificiais. Revista de Estudos Ambientais, v.15, n.2, p.48-57, 2013.

FIALHO, A. L. L.; SOUZA-CÁCERES, M. B.; SILVA, W. A. L.; ARRUDA, E. D. S.; KISCHEL, H.; RIBEIRO-FERREIRA, M. G. C.; MEDEIROS, C. F.; SILVA, J. R.; OLIVEIRA, M. V. M.; FERRAZ, A. L. J. Efeito do estresse térmico calórico agudo e crônico sobre a qualidade oocitária de bovinos de raças adaptadas. Arquivo Brasileiro de Medicina Veterinária e Zootecnia, v.70, n.1, p.64-72, 2018.

IBGE - INSTITUTO BRASILEIRO DE GEOGRAFIA E ESTATISTICA. Bases Cartográficas Contínuas. Rio de Janeiro: 2021. Disponível em:

https://www.ibge.gov.br/geociencias/cartas-e-mapas/bases-cartograficas-continuas.html

INMET - INSTITUTO NACIONAL DE METEOROLOGIA. BDMEP: Banco de Dados Meteorológicos para Ensino e Pesquisa. Brasília: 2021.

Disponível em: https://bdmep.inmet.gov.br/

MANTECA, X.; SILVA, C. A.; BRIDI, A. M.; DIAS, C. P. Bem-estar animal: conceitos e formas práticas de avaliação dos sistemas de produção de suínos. Revista Semina: Ciências Agrárias, v.34, n.6, p.42134230, 2013. 
NASH, J. E.; SUTCLIFFE, J. V. River flow forecasting through conceptual models. Part I: A discussion of principles. Journal of Hydrology, v.10, n.3, p.282-290, 1970.

OLIVEIRA, Z. B.; SILVA, C. M.; SOUZA, I. J.; LINK, T. T. Cenários de mudanças climáticas e seus impactos na produção leiteira no sul do Brasil. Brazilian Journal of Biosystems Engineering, v.12, n.2, p.110-121, 2018.

PEEL, M. C.; FINLAYSON, B. L.; MCMAHON, T. A. Updated world of the Köppen-Geiger climate classification. Hydrology and Earth System Sciences, Göttingen, v.11, n.5, p.1633-1644, 2007. DOI: https://doi.org/10.5194/hess-11-1633-2007

QGIS DEVELOPMENT TEAM. QGIS Geographic Information System. Open Source Geospatial Foundation Project. Versão 3.12.0-București. 2020.

RAMOS, H. C.; DALLACORT, R.; NEVES, S. M. A. S.; DALCHIAVON, F. C.; SANTI, A.; VIEIRA, F. F. Precipitação e temperatura do ar para o estado de Mato Grosso utilizando krigagem ordinária. Revista Brasileira de Climatologia, v.20, n.13, p.211-233, 2017.

R CORE TEAM. R: A language and environment for statistical computing. R Foundation for Statistical Computing, Viena, Austria. Versão 3.5., 2020.

SILVA, M.R.; SOUZA, B. B.; GUIMARÃES, L. J.; COSTA, D. F.; ROCHA, E. F.; SOUTO, D. V. O.; SILVA, E. M. N. Estresse térmico e sua influência na fisiologia hormonal de pequenos ruminantes. Journal of Animal Behaviour and Biometeorology, v.4, n.2, p.50-54, 2016.

THOM, E. C. The discomfort index. Weatherwise, Philadelphia, v.12, n.2, p.57-61, 1959. DOI: https://doi.org/10.1080/00431672.1959.9926960

VIANA, M. P.; MEDEIROS, A. R.; SOUZA, B. B. Efeitos do estresse térmico sobre a fisiologia, produção e reprodução de caprinos. Revista Agropecuária Científica no Semiárido, v.9, n.4, p.01-08, 2013.

WU, C. L.; CHAU, K. W.; FAN, C. Prediction of Rainfall Time Series Using Modular Artificial Neural Networks Coupled with Data-Preprocessing Techniques. Journal of Hydrology, v.389, n.1, p.146-167, 2010.

YASAR, A.; SIMSEK, E.; BILGILI, M.; YUCEL, A. Estimation of relative humidity based on artificial neural network approach in the Aegean Region of Turkey. Meteorology and Atmospheric Physics, v.115, n.1-2, p.81-87, 2012. 